\title{
Acknowledgment of Ad Hoc Reviewers
}

The Editor and Associate Editors wish to acknowledge the following individuals who reviewed four or more manuscripts for the Journal of Consulting and Clinical Psychology between October 1, 2015 and October 1, 2016 and were designated Principal Ad Hoc Reviewers in recognition of their service.

Timothy Mark Anderson
Daniel M. Bagner
Brian Borsari
Thomas Bradbury
Clara M. Bradizza
Jason Chapman
Jonathan S. Comer
Philip A. Cowan
Paul Crits-Christoph

Timothy Mark Anderson

\author{
George DuPaul \\ Catherine Flynt Eubanks \\ Steven W. Evans \\ Fredrik Falkenström \\ Norah C. Feeny \\ Zac Imel
}

Nikolaos Kazantzis

$\begin{array}{ll}\text { Helena C. Kraemer } & \begin{array}{l}\text { Megan E. Piper } \\ \text { Mark B. Powers }\end{array} \\ \text { Christina Lee } & \text { David Rosenfield } \\ \text { Bryce D. McLeod } & \text { Timothy Stickle } \\ \text { James G. Murphy } & \begin{array}{l}\text { Eric Wagner } \\ \text { Bruce E. Wampold }\end{array} \\ \text { Julie Sarno Owens } & \end{array}$

Linda Pfiffner

Ad Hoc Reviewers

The Editor and Associate Editors gratefully acknowledge the following ad hoc reviewers, who reviewed manuscripts for Journal of Consulting and Clinical Psychology between October 1, 2015 and October 1, 2016.

Benjamin Aas

Alexis D. Abernethy

Jonathan Abramowitz

Ana M. Abrantes

Erin Cecilia Accurso

Juliana Acosta*

Jenna Lauren Adamowicz

Abby Adler

Stewart Agras

Adrian Aguilera

Timothy Allen*

Brooke A. Ammerman

Paul C. Amrhein

Arthur Anastopoulos

Page Anderson

Gerhard Andersson

Gavin Andrews

Michael D. Anestis

Emily B. Ansell

Michael Antoni

Allison J. Applebaum

Alejandra Arango*

Caley Arzamarski*

Natalie Arbid*

Joan Asarnow

Jonathan Ashar*

Rebecca Ashare

Elizabeth Aston*

David C. Atkins

Marc S. Atkins

Randy Patrick Auerbach

Marleine Azar*

Kimberly Babson

Sudie Back

Christal L. Badour

John S. Baer

R. Michael Bagby

Amanda Baker

Scott Baldwin
Kimberly F. Balsam

Tom Baranowski

Margaret Rose Barlow*

Nancy Barnett

Miya Barnett

Thorsten Barnhofer

Paula Barrett

Marna S. Barrett

Brian Baucom

Katie Baucom

Stephanie Bauer

Stephanie S. Bauman

Steven R. H. Beach

Carolyn Becker

Eni Becker

Kathryn Becker-Blease

Michele Bedard-Gilligan

Rinad Beidas

Melanie Bennett

James Bennett-Levy

Lisa Benson*

Aaron Bergman*

Matthias Berking

Noah Chase Berman

Amit Bernstein

Ryan Beveridge

Vickie Bhatia

Jessica Black

Maureen M. Black

Rebecca Kate Blais

Carlos Blanco

Aaron Blashill

Erika Bloom

Tabatha Blount

Arthur W. Blume

Beth Bock

Marilisa Boffo*

Jessica Bomyea

Erin Bonar

Marcel Bonn-Miller
Marina Bornovalova

Joaquin Borrego

Jantine Boselie

James F. Boswell

Aaron J. Boulton

Sarah Bowen

Derrecka M. Boykin

Scott R. Braithwaite

Meagan Brem*

David A. Brent

Deborah Brief

Willoughby Britton

Rebecca L. Brock

Elissa Brown

Monique Brown*

Michael Browning

Steven E. Bruce

Steven M. Brunwasser

Alicia Bunger

Barbara J. Burns

Mark Burton*

Andrew Busch

Meghan Butryn

Susan Byrne

Jose Alonso Cabriales

Fary M. Cachelin

Sean Cahill

Catherine Caldwell-Harris

Jennifer L. Callahan

Judith Ann Callan

Laura Campbell-Sills

Derek Caperton*

Fawn Caplandies*

Dan Capron

Esteban Cardemil

James Caringi

Adam W. Carrico

Alice Carter

Jeffrey Carter
Mary Louise Cashel

Diane T. Castillo

Timothy A. Cavell

Alexander L. Chapman

Kathleen Chard

Jennifer S. Cheavens

Eunice Chen

JeeWon Cheong

Steve Chermack

Vien Cheung*

Amanda Chiapa*

Brian C. Chu

Harold Chui* $^{*}$

Anna Ciao

Patricia Cioe*

Joshua Clapp

David M. Clark

Mari L. Clements

Bonnie A. Clough

Rachael Coakley

Rebecca Cobb

Susan D. Cochran

Bryan N. Cochran

Scott F. Coffey

Josh Cohen*

Judy Cohen

Anahi Collado

Bruce Compas

Kevin Connolly

Joseph I. Constans

Ned Cooney

Zafra Cooper

James Cordova

Jesse Cougle

Linda Craighead

Giuseppe Craparo

Torrey A. Creed

Kasey Creswell

Ioana Alina Cristea

Ross D. Crosby 
Scott J. Crow

Susan L. Crowley

E. Mark Cummings

John Cunningham

John F. Curry

Maeve Cyr ${ }^{*}$

Sara Czaja

Lynnda Dahlquist

Sannisha Dale

Summer Danner*

BreAnne Allen Danzi

Elizabeth D'Amico

Louanne W. Davis

Christopher Davis

Peter de Jonge

Rianne de Kleine

Andres De Los Reyes

Brett J. Deacon

Blake Dear

Suzanne E. Decker

Kevin L. Delucchi

Kelly S. DeMartini

Aaron Dembe*

Ellen DeVoe

Carlo C. DiClemente

Sona Dimidjian

Thomas J. Dishion

Katherine A. Dondanville

Alex R. Dopp*

Brian D. Doss

Deborah Drabick

Cristiane Duarte

Matthew DuBois*

Patrick Dulin

Dustin Duncan

Robert Dvorak

Emily Dworkin*

Allison Earl

Nicholas Eaton

Christopher Eckhardt

Kamryn Eddy

Hester V. Eeren

Thomas Ehring

Caroline Ehrlich*

Maarten C. Eisma

David Ekers

JoAnna Elmquist*

Lisa Elwood

Robert E. Emery

Elissa Epel

Catherine C. Epkins

Jeff Epstein

Christopher Erbes

Denise B. Ernst

Deinera Exner-Cortens

Michael Ezenwa

Angela Fang

Lara J. Farrell

Brian Feinstein

Matthew T. Feldner

Thomas Fergus
Stephanie Fernandez*

Matt Field

Craig Field

David Fiellin

Charles Figley

John W. Finney

Peter L. Fisher

Skye Fitzpatrick $^{*}$

Mark S. Fleisher

Christoph Flückiger

Jason Fogler

Frederick Foley

Julian Ford

Rex L. Forehand

Greta Francis

Joseph C. Franklin

C. Laurel Franklin

Patricia Frazier

Steffany J. Fredman

Mark C. Freestone

David M. Fresco

Fred Friedberg*

Christopher Frueh

Daniel Fulford

Matthew Gallagher

Robert Gallop

Tara Galovski

Fabian Gander

Eric Garland

Shelia Garland

Scott Gaynor

John Geddes

Andrew L. Geers

Lois A. Gelfand

Pamela A. Geller

Andrew Gelman

Monica M. Gerber*

Alexandra Gibson*

Eva Gilboa-Schechtman

Jane Gillham

Catherine R. Glenn

Shirley M. Glynn

Susan Godley

Elizabeth M. Goetter

Andrea B. Goldschmidt

Joseph P. Gone

Jeffrey S. Gonzalez

Adam Gonzalez

Rafael A. Gonzalez

Marielle Goossens

Chanelle Gordon*

Kristina Coop Gordon

Amy Gorin

Suzanne Graham

Eric Granholm

Demond Grant

Paul Grant

Sean Grant

Shelly F. Greenfield

Joseph Greer

James Griffith

Amie Grills

Carlos M. Grilo
Martin Grosse Holtforth

Nancy Grote

John Gunderson

Hamish A. Gunn*

Kaya Gurak*

Kilem Li Gwet

David A. F. Haaga

Willie Hale*

Brian James Hall

Moira Haller

Kevin Hallgren

Nathan B. Hansen

R. Karl Hanson

Tae Hart

Allison G. Harvey

Elizabeth Harvey

Penelope Hasking

Christina Hassija

David Hawes

Alan Hawkins

Matthew Hawrilenko*

Sarah Hayes-Skelton

Ann Frances Haynos

Jeff Hecker

Thomas Heidenreich

Kimberly Hepner

Whitney Heppner

Reid Hester

Charmaine Higa

Anja Hilbert

Thomas Hildebrandt

Asle Hoffart

Jasara Hogan*

Aaron Hogue

Ryan Holliday

Grayson N. Holmbeck

Rolf Holmqvist

Amy Holtzworth-Munroe

Monica Webb Hooper

Debra Hope

Lindsey B. Hopkins

Keith Horvath

Adam O. Horvath

William Hoyt

Jennifer L. Hudson

David M. Huebner

Stanley Huey

Christopher Hughes*

David Huh

Jonathan D. Huppert

Nick S. Ialongo

Brooke Ingersoll

Karen S. Ingersoll

Jonathan Jackson

Nicholas C. Jacobson

Jolene Jacquart*

Lisa James

Beth Janis*

M. Kay Jankowski

Lisa Jaycox

Amanda Jensen-Doss
Amishi Jha

Yuan Jiang*

Matthew D. Johnson

Charlotte Johnston

Thomas Joiner

Hendree Jones

Alexander H. Jordan*

Christopher W. Kahler

Viktor Kaldo

Jennifer W. Kaminski

Carl Kantner*

Jennifer Katz

David Kealy

Susan Kelley

Eva Kemps

Laura Kiken

Dean Kilpatrick

Brian Kiluk

Hyoun K. Kim

Youngmee Kim

Rachel Kimerling

Drew Kingston

Katharina Kircanski

Daniel Kivlahan

Dennis M. Kivlighan

Evan Kleiman

Alexander Kline*

E. David Klonsky

Jacqueline D. Kloss

Lauren Knopf*

Laura Kohn-Wood

David J. Kolko

Alex Kopelowicz

Ernst Koster

Magdalena Kulesza

Janice Kuo

Matt G. Kushner

Ian Lambie

Sara Landes

Ariel J. Lang

Joshua M. Langberg

Jennifer LanghinrichsenRohling

Calvin M. Langton

Cathy Lau-Barraco

Sharon Lawn

Suzanne Lechner

Carol Lee*

Steve S. Lee

Robert F. Leeman

Ellen Leen-Feldner

Paul Lehrer

Carl Lejuez

Leslie Leve

Adam Leventhal

Michael Levin

Cheri Levinson

Hannah Levy*

Yan Leykin

Gabrielle Liverant

Marci Lobel

Nicholas Long 
Steven R. Lopez

Catherine Lord

Andrés Losada

Patrick Love*

Joan Luby

Sheng Luo

Wolfgang Lutz

Janet Lydecker

Aaron R. Lyon

Elizabeth P. MacKenzie

Daniel Mackin*

Colin MacLeod

Brent Mallinckrodt

Robert Maniglio

Jacob Manuel*

Jessica Maples

David K. Marcus

Marsha Marcus

Jurgen Margaf

Matthew P. Martens

Daniel Martin

Craig A. Mason

Greta M. Massetti

David Mataix-Cols

Amanda Mathew

Walter Matthys

Vickie M. Mays

James Mazza

Suzanne Mazzeo

Jennifer McCabe Beane

Michael R. McCart

Kevin Scott McCarthy

Elizabeth McCauley

Erin A. McClure

Barbara S. McCrady

Christina S. McCrae

Peter McEvoy

Lynn McFarr

Eleanor McGlinchey*

Susan McGurk

R. Kathryn McHugh

James R. McKay

Dean McKay

Katherine McMahon*

Robert J. McMahon

Cori McMahon

John R. McQuaid

Tara Mehta

Matthew Meisel ${ }^{*}$

Nadine M. Melhem

Jennifer Merrill

Lidia Meshesha*

Stephen Messer

Jane Metrik

Alicia E. Meuret

Andrea Meyer*

Ilan H. Meyer

Stephanie Milan

Kathrin Milbury

Mary Miller

Sara Milrad*

Mary Minges*

Jeanne Miranda
Damon Mitchell

Markus Moessner

Catherine Mogil

Jan Mohlman

Christine Molnar

Sherry Davis Molock

Michael T. Moore

Timothy E. Moore

Alina Morawska

Robert Morgan

Jace Morganstein*

David A. Moscovitch

Jason Moser

Catherine E. Mosher

Theresa Moyers

Ricardo F. Munoz

Simone Munsch

Christopher Murphy

Patricia Murphy

Sylvie Naar-King

Kristen Naragon-Gainey

Urs Nater

Ricarda Nater-Mewes*

Andrada Neacsiu

Clayton Neighbors

Michael Newcomb

Trang Nguyen

Sonya Norman

Danay Novoa*

Caoilte Ó Ciardha

Kieron O'Connor

Stephen O'Connor

Jessica O'Leary*

Suzanne O'Neill

Terje Ogden

Bunmi Olatunji

Allen M. Omoto

Holly K. Orcutt

Pamela Orpinas

Flávia L. Osório

Lars-Goran Ost

Michael Otto

Ed Pace-Schott

John Pachankis

Timothy F. Page

Steven C. Palmer

David W. Pantalone

George Papandonatos

Santiago Papini*

Lauren Papp

Justin Parent

Mallie J. Paschall

Freda Patterson

Susan Paxton

Matthew R. Pearson

Eric R. Pedersen

Tara S. Peris

Sean Perrin

J. Christopher Perry

Carol Peterson

Jeremy Pettit
Martha Phillips

Sean Phipps

Caitlin Pinciotti*

Matthew C. Podlogar

Antonio Polo

Alberta Pos

Patrick Pössel

Kyle Possemato

Bridget Poznanski*

Harry Prapavessis

Matthew Price

Holly G. Prigerson

Mark A. Prince

Ron Prinz

James Prochaska

Amy Przeworski

Christina Psaros

Nicole D. Pukay-Martin

Erdem Pulcu*

Sarah M. Rabbitt

Sarah E. Racine

Bethany Raiff

Rajeev Ramchand

Fabian Ramseyer

Ron Rapee

Sheila Rauch

Jennifer P. Read

Oakleigh Reed ${ }^{*}$

Megan Renna*

Fritz Renner

D’Arcy Reynolds

Mark A. Richardson

Ty Ridenour

Traci R. Rieckmann

Kira Riehm*

Shireen Rizvi

Donald Robinaugh

John Robinson

Rachel F. Rodgers

Craig Rodriguez-Seijas*

Lizabeth Roemer

Joshua Rooks*

Craig Rosen

David H. Rosmarin

Alex Rothbaum*

Barbara Olasov Rothbaum

Cindy L. Rowe

Lorelei Simpson Rowe

Julian Rubel*

Miriam Rubenson*

Avinadav Rubin*

Brittany Rudd*

Michael Russell

Josef I. Ruzek

Richard Ryan

Ehri Ryu

Ebru Salcioglu

Kristi Salters-Pedneault

Amanda Sanchez*

Irwin N. Sandler

Shannon Sauer-Zavala

Josee Savard
Gauri Saxena

Steven L. Sayers

Cindy M. Schaeffer

Christine D. Scher

Henrike Schlagert

Victoria Schlaudt*

Ricarda Schmidt*

Sophie Schneider*

Alexander Schoemann

Stephen Schueller

Julie Schumacher

Zev Schumann-Olivier

Jeremiah A. Schumm

Karen T. G. Schwartz*

David C. Schwebel

Forrest Scogin

Meredith Sears*

Zindel Segal

Chris Segrin

Edward Selby

Laura Seligman

Katrina Selverian*

Julianne Serovich

Kristen Serowick*

Heather Shaw

Daniel Shaw

Katherine Shear

Lisa Sheeber

Allen C. Sherman

Ashley Hampton Shields

Stephen R. Shirk

Margaret H. Sibley

Scott D. Siegel

Matthew Skinta

Mia Skytte-O'toole*

Rachel Small*

Amy M. Smith Slep

Denise M. Sloan

Brandy Smith*

Tristram Smith

Joshua M. Smyth

Mark Sobell

Kathryn Soltis*

Tina Soma*

Vanessa Somohano*

Susan South

Susan Sprich

Lexine Stapinski

Cynthia A. Stappenbeck

Tyrel Starks

Emily Stasko*

Shari Steinman

Kyle R. Stephenson

Sunita M. Stewart

Eric A. Storch

Timothy Joseph Strauman

Amy E. Street

Randi Streisand

Daniel R. Strunk

Gregory L. Stuart

Lourdes Suarez-Morales

Kieran Sullivan

Michael K. Suvak

Rachel Sweenie* 
Robyn Sysko

Darius Tandon

Emily Tanner-Smith

Michael Tarren-Sweeney

Heather N. Taussig

Tonya Tavares*

Charles Taylor

Daniel J. Taylor

Bethany Teachman

Michael J. Telch

Michael E. Thase

Brett D. Thombs

Renee Thompson

Tim Thornberry

Stacey S. Tiberio

David Tolin

Edil Torres-Rivera

Olivia Tousignant-Pienkos*

Lara Traeger

Giao Q. Tran

Kimberli Treadwell

Christopher J. Trentacosta

Hugo Trevisi*

Hester Trompetter

Elisa Trucco
Wolfgang Tschacher

Aaron P. Turner

Erick Turner

J. Blake Turner

David P. Valentiner

Wim Van den Noortgate

Michael Vanderlind

Maarten Vansteenkiste

Rachel Vaughn-Coaxum*

Tine Vervoort

David Victorson

Desirae Vidaurri*

Roger Vilardaga

Jeffrey R. Vittengl

Ana Maria Vranceanu

Jennifer Wachen

Thomas A. Wadden

Anne C. Wagner

Milton Wainberg

Glenn Waller

Erin Walsh

Jennifer Walsh

Scott Walters

Jason J. Washburn
Christian A. Webb

Thomas L. Webb

Florian Weck

Risa B. Weisberg

Amy G. Weisman de Mamani

Mark Weist

Henny A. Westra

Elizabeth Westrupp

Julie Wetherell

Mark A. Whisman

Helene White

Janis L. Whitlock

Jelte Wicherts

Reinout Wiers

Jennifer Wildes

Joshua F. Wiley*

Victoria M. Wilkins

Sylia Wilson

Stephen Wilson

Shannon Wiltsey Stirman

Rena Wing

Blair Wisco

Katie Witkiewitz

Erika Wolf

Caitlin Wolford-Clevenger*

Kate Wolitzky-Taylor
Stephen Wonderlich

Jennifer Wong

Jeffrey Wood

Erica Woodin

Aidan Wright

Til Wykes

Shu Xu

Chelsie Young*

Jami Young

John Young

Eric A. Youngstrom

William Yule

Dina Zalaznik*

Emily Zale*

Alyson Kay Zalta

Julia Zeroth*

Sigal Zilcha-Mano

Shelby Zuckerman*

Lucas Zullo*

Michael Zvolensky

* Denotes co-reviewers who severed under the supervision of a primary reviewer. 